\title{
Higgs doublet as a Goldstone boson in perturbative extensions of the Standard Model
}

\author{
Brando Bellazzini ${ }^{a}$, Stefan Pokorski ${ }^{b}, V$ yacheslav S. Rychkov ${ }^{a, b}$ \\ Alvise Varagnolo ${ }^{a, c}$ \\ a Scuola Normale Superiore and INFN, Piazza dei Cavalieri 7, I-56126 Pisa, Italy \\ ${ }^{b}$ Institute of Theoretical Physics, Warsaw University, Hoza 69, 00-681 Warsaw, Poland \\ ${ }^{c}$ Dip. di Fisica, Univ. di Roma La Sapienza, P.le A. Moro, 2, I-00185 Rome, Italy
}

\begin{abstract}
We investigate the idea of the Higgs doublet as a pseudo-Goldstone boson in perturbative extensions of the Standard Model, motivated by the desire to ameliorate its hierarchy problem without conflict with the electroweak precision data. Two realistic supersymmetric models with global $S U(3)$ symmetry are proposed, one for large and another for small values of $\tan \beta$. The two models demonstrate two different mechanisms for EWSB and the Higgs mass generation. Their experimental signatures are quite different. Our constructions show that a pseudo-Goldstone Higgs doublet in perturbative extensions is just as plausible as in non-perturbative ones.
\end{abstract}




\section{Introduction}

The idea of the Higgs doublet as a pseudo-Goldstone boson of some extended global symmetry has been proposed to ameliorate the hierarchy problem of the Standard Model (SM) [1]. Usually, it is linked to a new strongly interacting sector, responsible for spontaneous breaking of the global symmetry [2], 3], 4]. There are interesting signatures of this idea, among others related to the unitarization procedure in WW scattering [5],[6]. However, there are strong constraints on the scale $f$ of the spontaneous breaking of global symmetry of the strong sector. Low values of $f$, say $f \lesssim 500 \mathrm{GeV}$, cannot be easily reconciled with electroweak precision tests and B-physics data [7, while larger $f$ reintroduces the hierarchy problem with the required finetuning growing a: 1 $(f / v)^{2}$. So, in practice, models of this kind do not avoid certain tension.

There is some room for the idea of the Higgs doublet as a pseudo-Goldstone boson in perturbative extensions of the SM as well, with global symmetry broken in the perturbative regime. In general, one may expect such perturbative models to avoid excessive finetuning in the ElectroWeak Symmetry Breaking (EWSB) sector with no conflict with the electroweak precision data, generic for non-perturbative models. This possibility has been discussed in non-supersymmetric [7, [8] and supersymmetric [9, [10, 11, 12, 14, 113, models, however for various reasons those models are not fully satisfactory. In the present paper we explore it further in supersymmetric (SUSY) models with extended global symmetry of the Higgs sector. We discuss two models which differ in various respects and illustrate various aspects of the general approach. As global symmetry we take $S U(3)$, the minimal one that can give Higgs doublet as a Goldstone boson in SUSY.

The first model (Model I) remains perturbative up to the GUT scale. The global symmetry and the electroweak symmetry are broken by radiative corrections to the mass parameters, generated by a large Yukawa coupling, similarly to the Minimal Supersymmetric Standard Model (MSSM) (for earlier attempts, see [11]). Stabilization of the global symmetry breaking scale $f$ can be achieved by quartic scalar coupling in large $\tan \beta$ regime. The model relies on the "double protection" mechanism, where the interplay between supersymmetry and an approximate global symmetry forbids the quadratic higgs term to receive a large logarithmic contribution from the UV cutoff $\Lambda \sim M_{\text {GUT }}$ which is actually replaced by the scale of global symmetry breaking $f$. Thus one may hope to get, for the same values of stop mass, much less finetuning than in the MSSM. The values $f \gtrsim 2 \mathrm{TeV}$ minimize finetuning of the model, while at the same time allowing the physical Higgs boson mass above the experimental bound of $115 \mathrm{GeV}$. Phenomenology of the Higgs sector of the model is very similar to the decoupling regime of the MSSM. In particular, for $f \gtrsim 2 \mathrm{TeV}$ the WW scattering is unitarized almost completely by the lightest Higgs boson. The model is however distinguished by the presence of a relatively light doubly-charged Higgsino.

In our second example (Model II) supersymmetry provides a consistent framework for stabilizing the minimum of the global symmetry breaking. It is a supersymmetric version of the approach to the EWSB proposed in Ref. [7], with the breaking driven by a tadpole of the $S U(2) \times U(1)$ singlet component of the full scalar multiplet. An interesting point about this mechanism is that this tadpole, while being linear in the fundamental field, generates the Higgs quartic when the $\sigma$-model structure is taken into account. This quartic dominates the usual D-term quartic at low $\tan \beta$, so that the physical Higgs mass is determined by the soft SUSY breaking terms only. Modell II has a very different phenomenology with respect to MSSM since it allows for low $f$. However, it needs

\footnotetext{
${ }^{1}$ Here and throughout the paper $v$ is the electroweak scale in $v \simeq 174 \mathrm{GeV}$ normalization.
} 
an UV completion at a scale $O(20 \mathrm{TeV})$, where the SUSY model becomes strongly interacting. In both models finetuning is $O(10 \%)$.

\section{Model I}

In the MSSM, the lightest Higgs boson mass is determined by the effective quartic coupling, which depends logarithmically on the stop mass. Large $\tan \beta$ is then favored, to minimize the value of the stop mass consistent with the experimental bound $m_{h}>115 \mathrm{GeV}$, and the finetuning in the Higgs potential. The latter is proportional to $m_{\tilde{t}}^{2} \log \Lambda$ and for $\Lambda \sim M_{\mathrm{GUT}}$ remains, unfortunately, of order of $1 \%$.

The model we propose in this section retains the MSSM correlation between the stop mass and the Higgs boson mass, thus also requiring large $\tan \beta$ for reasonable values of $m_{\tilde{t}}$. However, it is based on the idea of the double protection of the Higgs potential [10, [11] and gives, for the same values of $m_{\tilde{t}}$, factor 10 less finetuning than MSSM.

We begin with the effective model below certain scale $F$ based on the symmetry $S U(2)_{L} \times U(1)_{y}$, where $S U(2)_{L}$ is gauged subgroup of a global $S U(3)$. Its UV completion (above $F$ ) can be similar to that of [11], and we will return to it below. The $S U(3)$-symmetric Higgs sector consists of a triplet $\mathcal{H}_{d}$ and an antitriplet $\mathcal{H}_{u}$, while the chiral fermion multiplets of the top sector are the triplet $\Psi=(Q, T)^{T}$ and quark singlets $t^{c}$ and $T^{c}$.

Under $S U(2)_{L} \times U(1)_{y}$ the triplets split into doublets $H_{u, d}$ and singlets $S_{u, d} 2$

$$
\mathcal{H}_{d}^{T}=\left(H_{d}^{T}, S_{d}\right), \quad \mathcal{H}_{u}=\left(H_{u}, S_{u}\right) .
$$

We look for a model in which the global $S U(3)$ is spontaneously broken by vacuum expectation values $(\mathrm{VEVs})$ aligned so that $S U(2)_{L} \times U(1)_{y}$ gauge symmetry remains unbroken:

$$
\mathcal{H}_{d}^{T}=\left(0,0, f_{d}\right), \quad \mathcal{H}_{u}=\left(0,0, f_{u}\right),
$$

and $\tan \beta \equiv f_{u} / f_{d}$ is large. We shall assume that the soft term mass scale $m_{\text {soft }} \sim f \ll F$. The minimum of the $S U(3)$-symmetric scalar potential at large $\tan \beta$ generically requires negative $\mathcal{H}_{u}$ mass squared, which leads to runaway directions, unless there is a stabilization mechanism. Stabilization by D-terms of some, e.g. $U(1)$, gauge interactions is not a satisfactory mechanism [10], while stabilization by the quartic coupling $\left|\mathcal{H}_{u}\right|^{4}$ is constrained by the holomorphicity of the superpotential.

With two Higgs triplet chiral superfields, the minimal field content leading to stabilization by the quartic consists of two symmetric tensors $\mathcal{Z}_{1}$ and $\mathcal{Z}_{2}$,

$$
\mathcal{Z}_{i}=\left(\begin{array}{c|c}
T_{i} & H_{i} / \sqrt{2} \\
\hline H_{i}^{T} / \sqrt{2} & z_{i}
\end{array}\right)
$$

Here the $T_{i}$ 's are $S U(2)$ triplets with $Y_{1,2}= \pm 1, H_{i}$ 's are doublets with $Y_{1,2}= \pm 1 / 2$, and $z_{i}$ 's are singlets. The superpotential of our model reads (in the following we anticipate large $\tan \beta$ solution)

$$
W=\lambda \mathcal{Z}_{2} \mathcal{H}_{u} \mathcal{H}_{u}+\mu \mathcal{H}_{u} \mathcal{H}_{d}+\mu_{Z} \mathcal{Z}_{1} \mathcal{Z}_{2}+y \mathcal{H}_{u} \Psi t^{c}+m T^{c} T
$$

\footnotetext{
${ }^{2}$ It's useful to keep in mind that our doublet $H_{u}$ is related to the MSSM doublet $H_{u}$ via $H_{u \text {.our }}=\varepsilon \cdot H_{u, \text { MSSM }}$.
} 
The last term breaks the global $S U(3)$ explicitly. It can originate from a UV completion as in [11. The scalar potential reads 3

$$
\begin{aligned}
V & =\left|\lambda \mathcal{H}_{u} \mathcal{H}_{u}+\mu_{Z} \mathcal{Z}_{1}\right|^{2}+\left|2 \lambda \mathcal{Z}_{2} \mathcal{H}_{u}+\mu \mathcal{H}_{d}\right|^{2}+\mu_{Z}^{2}\left|\mathcal{Z}_{2}\right|^{2}+\mu^{2}\left|\mathcal{H}_{u}\right|^{2}+V_{\text {soft }}, \\
V_{\text {soft }} & =m_{d}^{2}\left|\mathcal{H}_{d}\right|^{2}+m_{u}^{2}\left|\mathcal{H}_{u}\right|^{2}+m_{Z 1}^{2}\left|\mathcal{Z}_{1}\right|^{2}+m_{Z 2}^{2}\left|\mathcal{Z}_{2}\right|^{2}-\left(m_{3}^{2} \mathcal{H}_{d} \mathcal{H}_{u}+\text { H.c. }\right) .
\end{aligned}
$$

The soft terms in (2.2) depend on their initial values at the GUT scale and on the renormalization group (RG) running in the $S U(3)$-symmetric theory. We expect that the stop contribution will drive $m_{u}^{2}$ to negative values (while $m_{d}^{2}, m_{Z 1, Z 2}^{2}>0$ ), and global $S U(3)$ is spontaneously broken. Minimizing the potential for small $m_{3}^{2}$ (see Appendix @ for the running of $m_{3}^{2}$ ) and assuming $S U(3)$ to be broken in the $S U(2)$ singlet direction, we get

$$
\begin{aligned}
& \left\langle\left|\mathcal{H}_{u}\right|^{2}\right\rangle \equiv f_{u}^{2} \simeq-\frac{\mu_{u}^{2}}{2 \lambda_{\text {eff }}^{2}} \\
& \frac{\left\langle\left|\mathcal{H}_{u}\right|\right\rangle}{\left\langle\left|\mathcal{H}_{d}\right|\right\rangle} \equiv \tan \beta \simeq \frac{\tilde{\mu}^{2}+m_{d}^{2}}{m_{3}^{2}} \gg 1 \\
& \left\langle\left|\mathcal{Z}_{1}\right|\right\rangle \equiv f_{Z 1} \simeq-\frac{\lambda \mu_{Z} f_{u}^{2}}{\mu_{Z}^{2}+m_{Z 1}^{2}} \\
& \left\langle\left|\mathcal{Z}_{2}\right|\right\rangle \equiv f_{Z 2} \simeq-\frac{2 \lambda \mu f_{u} f_{d}}{\mu_{Z}^{2}+m_{Z 2}^{2}+4 \lambda^{2} f_{u}^{2}}
\end{aligned}
$$

where

$$
\begin{aligned}
\mu_{u}^{2} & =m_{u}^{2}+\mu^{2}<0 \text { (by assumption) } \\
\lambda_{\text {eff }}^{2} & =\lambda^{2} \frac{m_{Z 1}^{2}}{\mu_{Z}^{2}+m_{Z 1}^{2}} \\
\tilde{\mu}^{2} & =\mu^{2} \frac{m_{Z 2}^{2}+\mu_{Z}^{2}}{\mu_{Z}^{2}+m_{Z 2}^{2}+4 \lambda^{2} f_{u}^{2}}
\end{aligned}
$$

The $S U(3)$ is broken dominantly by $f_{u}$ and $f_{Z 1}$, with $f_{d}$ and $f_{Z 2}$ suppressed by large $\tan \beta$. Relative contribution of $f_{Z 1}$ versus $f_{u}$ decreases for smaller $\lambda$ and $\mu_{Z}$. The maximal value of $\lambda$ at the Fermi scale is constrained by the requirement of remaining perturbative up to the GUT scale (see Appendix $\mathrm{A}$ for the discussion of $\lambda$ running); we choose $\lambda=0.2$ in the following. The mass parameter $m_{u}^{2}$ gets $S U(3)$-symmetric negative contributions proportional to the Yukawa coupling $y$ and the coupling $\lambda$ (see Appendix Ad). In the following we will discuss the constraints on the parameter range following from the demand of no excessive finetuning in the potential for the $S U(3)$ breaking.

Spontaneous global $S U(3)$ breaking leads to five Goldstone bosons: an $S U(2)_{L}$ doublet $H$ and a real singlet $\eta$. The $H$ plays the role of the SM Higgs doublet. The singlet $\eta$ will not play any role in the following discussion; we will comment on its parametrization and physical effects below. For large $\tan \beta$ the Goldstones reside to a good approximation in the $\mathcal{H}_{u}$ and $\mathcal{Z}_{1}$. Up to terms of

\footnotetext{
${ }^{3}$ The omitted soft terms $\mathcal{Z}_{1} \mathcal{Z}_{2}$ and $\mathcal{Z}_{2} \mathcal{H}_{u} \mathcal{H}_{d}$ will in general be produced by running from the GUT scale; we have checked that their typical generated values are small and do not affect the dynamics.
} 
higher order in $H$, we have the following parametrization for the Goldstone bosons:

$$
\begin{aligned}
H_{d} & \simeq \alpha_{d} H, \quad H_{u} \simeq \alpha_{u} H^{\dagger} \\
H_{1} & \simeq \alpha_{Z 1} H^{\dagger}, \quad H_{2} \simeq \alpha_{Z 2} H \\
T_{1} & \simeq \frac{f_{Z 1}}{f^{2}} H^{\dagger} H^{\dagger}, \quad T_{2} \simeq \frac{f_{Z 2}}{f^{2}} H H \\
S_{u, d} & \simeq f_{u, d}, \quad z_{1,2} \simeq f_{Z 1, Z 2},
\end{aligned}
$$

where

$$
\begin{aligned}
\alpha_{u, d} & =f_{u, d} / f, \quad \alpha_{Z i}=\sqrt{2} f_{Z i} / f, \\
f^{2} & =f_{u}^{2}+f_{d}^{2}+2 f_{Z 1}^{2}+2 f_{Z 2}^{2} .
\end{aligned}
$$

In this parametrization $H$ has canonical kinetic term. As we will see later, experimental limit on the Higgs mass requires $f_{Z 1, Z 2} \ll f_{u} \sim f$.

The global $S U(3)$ is explicitly broken by the last term in the superpotential (2.1) and by the D-terms of $S U(2) \times U(1)$. Both terms contribute to the potential for the Goldstone boson $H$ :

$$
V=\delta m_{H}^{2}\left|H_{u}\right|^{2}+\left(\lambda_{0}+\delta \lambda\right)\left|H_{u}\right|^{4}+\ldots
$$

where the $\delta m_{H}^{2}$ and $\delta \lambda$ are obtained from the one-loop effective potential and $\lambda_{0}$ comes from the D-terms. We first discuss the effective potential contribution as it is responsible for the VEV of $H$ and the EWSB by the top-stop loops. Diagonalizing the top mass matrix, for large $\tan \beta$ we find (we introduce dimensionless coupling $\tilde{y}, m=\tilde{y}\left\langle\left|S_{u}\right|\right\rangle$ ):

$$
\begin{aligned}
& m_{t}=y_{t}\left\langle\left|H_{u}\right|\right\rangle, \quad y_{t} \equiv \frac{y \tilde{y}}{\sqrt{y^{2}+\tilde{y}^{2}}}, \\
& m_{T}=\left\langle\left|S_{u}\right|\right\rangle \sqrt{y^{2}+\tilde{y}^{2}} .
\end{aligned}
$$

For the couplings $y$ and $\lambda$ to remain perturbative up to the GUT scale (see Appendix), we need $y \lesssim 1.2$. Since $y_{t} \simeq 1$ (for $\left\langle\left|H_{u}\right|\right\rangle \simeq v$ ) we get $\tilde{y} \gtrsim 1.8$ and $m_{T} \gtrsim 2.2\left\langle\left|S_{u}\right|\right\rangle$, somewhat stronger than the theoretical lower bound $m_{T}=2 y_{t}\left\langle\left|S_{u}\right|\right\rangle$ realized for $y \simeq \tilde{y} \simeq \sqrt{2}$.

To realize the double protection mechanism, we assume that soft stop masses are $S U(3)$ symmetric at the scale $F$. To compute the effective potential, we assume for simplicity that these masses are universal:

$$
m_{Q}^{2}\left(|\tilde{Q}|^{2}+\left|\tilde{T}^{c}\right|^{2}+\left|\tilde{t}^{c}\right|^{2}\right)
$$

and we also neglect the possible left-right stop mixing. As in ref. [11] we get the following result:

$$
\delta m_{H}^{2}=-\frac{3}{8 \pi^{2}} y_{t}^{2}\left[m_{Q}^{2} \ln \left(1+\frac{m_{T}^{2}}{m_{Q}^{2}}\right)+m_{T}^{2} \ln \left(1+\frac{m_{Q}^{2}}{m_{T}^{2}}\right)\right]+\Delta,
$$

where

$$
\Delta \supset \frac{3 g_{2}^{2} M_{2}^{2}+g_{y}^{2} M_{y}^{2}}{8 \pi^{2}} \ln \frac{F}{M_{\text {soft }}}
$$


the contribution due to $S U(2) \times U(1)_{y}$ gauginos with soft masses $M_{2}, M_{y}$. At the same time the dominant contribution to $\delta \lambda$ is given by

$$
\delta \lambda \simeq \frac{3}{16 \pi^{2}} y_{t}^{4}\left[\ln \frac{m_{Q}^{2}}{m_{t}^{2}(1+x)}-2 x \ln (1+1 / x)\right], \quad x=m_{Q}^{2} / m_{T}^{2} .
$$

Notice that this correction is smaller than the corresponding MSSM correction for the same value of the stop mass, which is due to the negative contribution of the heavy $T$ quark. In the $m_{T} \gg m_{Q}$ limit, which will turn out to be relevant below, we recover the standard MSSM equations, with the important difference that the scale of the logarithm in (2.6) is given by $m_{T}$ instead of $M_{\mathrm{GUT}}$.

The D-term potential reads:

$$
V_{D}=\frac{g^{2}+g_{y}^{2}}{8}\left(\left|H_{u}\right|^{2}-\left|H_{d}\right|^{2}+\left|H_{1}\right|^{2}-\left|H_{2}\right|^{2}\right)^{2}=\frac{g^{2}+g_{y}^{2}}{8}\left(\alpha_{u}^{2}-\alpha_{d}^{2}+\alpha_{Z 1}^{2}-\alpha_{Z 2}^{2}\right)^{2}|H|^{4} .
$$

For the Higgs boson mass we get the following result:

$$
m_{h}^{2} \simeq\left(1-v^{2} / f^{2}\right)\left[M_{Z}^{2}\left(\alpha_{u}^{2}-\alpha_{d}^{2}+\alpha_{Z 1}^{2}-\alpha_{Z 2}^{2}\right)^{2}+4 \delta \lambda \alpha_{u}^{4} v^{2}\right] .
$$

The overall suppression factor is due to the $\sigma$-model correction to the wavefunction normalization of the Higgs doublet; it can be derived by keeping track of terms higher order in $H$ which were omitted in (2.4). Considering the large $\tan \beta$ suppression resulting in $\alpha_{d}$ and $\alpha_{Z 2}$ going to zero, we see that for a given $\delta \lambda$ the Higgs boson mass is maximized for

$$
\begin{gathered}
\tan \beta \rightarrow \infty, \quad \alpha_{Z 1} \rightarrow 0, \quad f \rightarrow \infty \\
m_{h}^{\max }=\left(M_{Z}^{2}+4 \delta \lambda v^{2}\right)^{1 / 2} .
\end{gathered}
$$

Expanding in the small negative corrections appearing when these parameters deviate from their optimal values, (2.9) can be numerically parametrized as follows

$$
m_{h} \simeq m_{h}^{\max }-1 \mathrm{GeV}\left[\left(\frac{12}{\tan \beta}\right)^{2}+\left(\frac{1.3 \mathrm{TeV}}{f}\right)^{2}+\left(\frac{\alpha_{Z 1}}{0.15}\right)^{2}\right]
$$

where the first and the second corrections come from finite values of $\tan \beta$ and $f$ respectively, and the third from a nonzero $\alpha_{Z 1}$. Since $m_{h}^{\max }$ cannot be much above $115 \mathrm{GeV}$ without a significant increase in finetuning (see Figs 1, 2 below), we should not allow the total loss in (2.12) to exceed $1 \div 2 \mathrm{GeV}$, which implies obvious constraints on the relevant parameters.

We now discuss the results for the Higgs boson mass and estimate the level of finetuning. In Fig. 1 we plot the Higgs boson mass (2.11) (i.e. without negative corrections given in (2.12)) as a function of $m_{Q}$ and $m_{T}$, using $\delta \lambda$ from eq. (2.8) with $m_{t}=172 \mathrm{GeV}$. Similarly to the MSSM, the Higgs boson mass increases with $m_{Q}$. For a fixed $m_{Q}$, the correction is maximized in the $m_{T} \gg m_{Q}$ limit.

In Fig. 2 we plot the finetuning in the Higgs mass term $m^{2}|H|^{2}$ which is needed to compensate the top-stop contribution (2.6):

$$
\mathrm{FT}_{1}=\frac{\left.\delta m_{H}^{2}\right|_{\Delta=0}}{m_{h}^{2} / 2}
$$




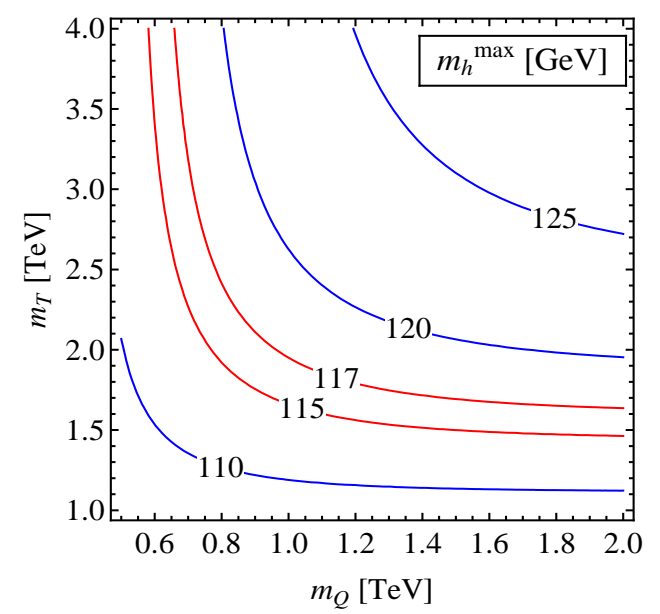

Figure 1: The maximal Higgs boson mass (2.11) as a function of $m_{Q}$ and $m_{T}$, see the text.

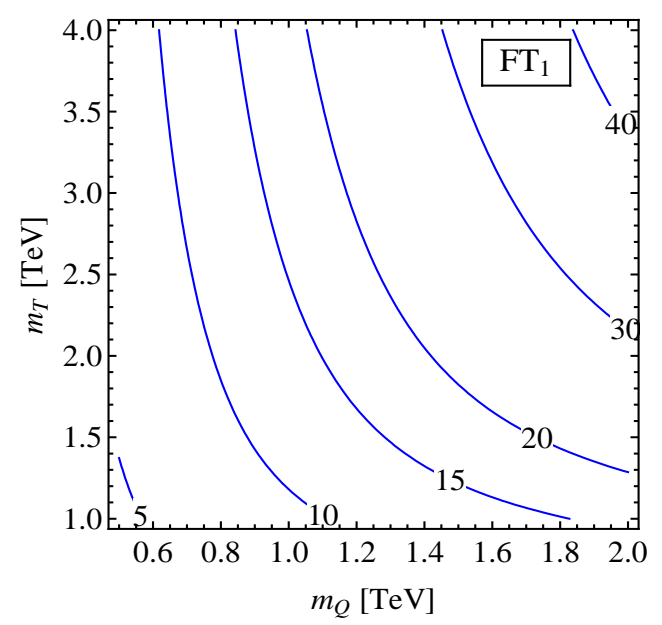

Figure 2: Finetuning (2.13) in the Higgs mass parameter needed to compensate for the top-stop loop contribution (2.6). 


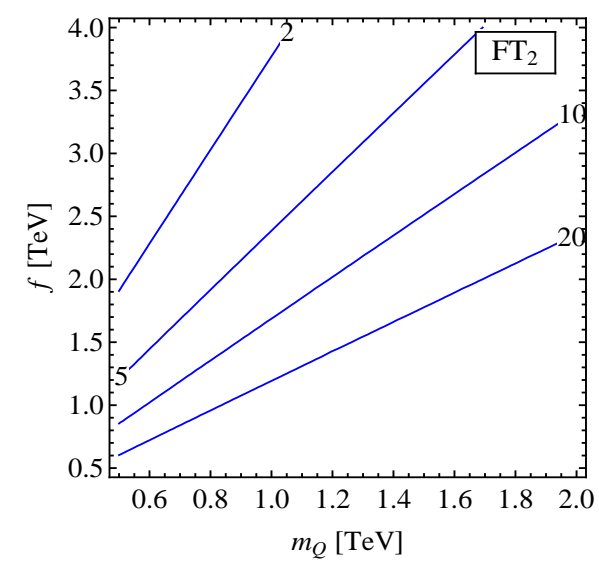

Figure 3: Finetuning (2.14) in the $S U(3)$-symmetric mass parameter $m_{u}^{2}$, see the text.

This finetuning increases quadratically with $m_{Q}$, but grows only logarithmically with $m_{T}$. Comparing Fig. 11 with Fig. 2, we see that $m_{Q} \sim 800 \mathrm{GeV}$ and $m_{T} \gtrsim 2.5 \mathrm{TeV}$ give $m_{h}>115 \mathrm{GeV}$ with about $10 \%$ finetuning $\left(\mathrm{FT}_{1}=10\right)$. Soft stop masses as small as $m_{Q} \sim 600 \mathrm{GeV}$ are possible provided that $m_{T}$ is raised up to $4 \mathrm{TeV}$.

Another source of finetuning in Model I is the $S U(3)$-symmetric top-stop contribution to the $m_{u}^{2}$ parameter of the scalar potential (2.2), which by (2.3) should not exceed $2 \lambda^{2} f_{u}^{2}$. The corresponding finetuning parameter

$$
\mathrm{FT}_{2}=\delta m_{u, s t o p}^{2} /\left(2 \lambda^{2} f^{2}\right)
$$

is plotted in the $m_{Q}-f$ plane in Fig. 3. where we assume $y \simeq 1, \lambda=0.2$. We see that this finetuning is less than $20 \%\left(\mathrm{FT}_{2}<5\right)$ for $m_{Q} \sim 800 \mathrm{GeV}$ and $f \gtrsim 2 \mathrm{TeV}$, which however translates into $m_{T}$ above $4 \mathrm{TeV}$.

In general raising $f$ (and $m_{T} \simeq 2 f$ ) we eliminate $\mathrm{FT}_{2}$ while $\mathrm{FT}_{1}$ grows only logarithmically. Unfortunately, in this limit the heavy top quark becomes undiscoverable at the LHC, and the scalar spectrum of the model resembles the standard MSSM at large $\tan \beta$ in the decoupling limit. In this case the only significant difference from the MSSM is the presence in the low-energy spectrum of states described by the tensors $\mathcal{Z}_{1}$ and $\mathcal{Z}_{2}$, i.e. triplets $T_{i}$, doublets $H_{i}$ and singlets $z_{i}$. The triplets contain doubly $\left(\tilde{T}_{1}^{++}\right.$or $\left.\tilde{T}_{2}^{--}\right)$and singly $\left(\tilde{T}_{1}^{+}\right.$or $\left.\tilde{T}_{2}^{-}\right)$charged and neutral $\left(\tilde{T}_{1}^{0}\right.$ or $\left.\tilde{T}_{2}^{0}\right)$ higgsinos and their scalar partners. Doublets $H_{i}$ have the same composition as the Higgs chiral superfields $H_{u, d}$. All those fields have common supersymmetric mass parameter $\mu_{Z}$. It enters in the equation (2.3) for the VEV of $z_{1}$ and is constrained by the requirement that no large negative $\alpha_{Z 1}$-correction to the Higgs boson mass should be present in Eq. (2.12). Assuming that all scalar soft masses are of the same order:

$$
M_{\mathrm{SUSY}} \sim m_{u} \sim \sqrt{2} \lambda f \sim 0.3 f,
$$

$\mu_{Z}$ is bounded by

$$
\mu_{Z} \lesssim \alpha_{Z 1} m_{\text {soft }} \lesssim f / 20
$$

\footnotetext{
${ }^{4}$ The formula given in [1] contains an extra $+3 / 2$ term in square brackets. Our formula is correct provided that $\delta \lambda$ is defined as the coefficient in the Higgs mass correction formula, Eq. (2.9).
} 
This estimate, while being subject to significant uncertainty, does indicate that the masses of new fermions, in particular of the doubly charged Higgsinos, are expected to be below $200 \div 300 \mathrm{GeV}$ even for $f$ as high as $2 \mathrm{TeV}$. We shall return to the phenomenological issues at the end of this section.

Finally, we need to comment on several other issues which are important for the consistency of our model. First, we note that the model can be UV completed as in Ref. [11], with the gauge group $S U(3) \times U(1)_{x}$ broken to the electroweak $S U(2) \times U(1)_{y}$ at the scale $F$. An extra pair of triplets $\Phi_{U, D}$ is responsible for this breaking, so that the full global symmetry of the scalar potential is $S U(3) \times S U(3)$. The $S U(3)$-breaking term $m T^{c} T$ in the Model I superpotential (2.1) originates naturally from an $S U(3)$-symmetric term

$$
y_{1} \Phi_{U} \Psi T^{c}, \quad y_{1} \sim m / F=\tilde{y} f / F,
$$

of the UV completed superpotential. As explained in [11], the soft mass terms of the $\Phi_{U}$ and $\Phi_{D}$ fields have to be very nearly universal at $F$, since their difference $m_{D}^{2}-m_{U}^{2}$ contributes to the mass term of the Higgs doublet via D-terms. Even assuming that these masses are universal at the GUT scale, superpotential interaction (2.15) will contribute to the running of $m_{U}^{2}$, so that at $F$ the masses will be split by

$$
m_{D}^{2}-m_{U}^{2} \simeq \frac{3 y_{1}^{2}}{8 \pi^{2}}\left(m_{Q}^{2}+m_{T^{c}}^{2}\right) \ln \frac{M_{\mathrm{GUT}}}{F}
$$

This contribution must be $\lesssim v^{2}$ which can be achieved by choosing $F \gtrsim 10 f$ as can be seen from the second eq. in (2.15).

On the other hand the $F$ scale cannot be too high because of the gaugino contributions to the Higgs mass, Eq. (2.7).

For completeness we have to say a few words about the 5th Goldstone boson $\eta$, a gauge singlet axion, which appears in addition to the Higgs doublet when $S U(3)$ is broken spontaneously to $S U(2)$, as already mentioned above. This Goldstone is associated with a global $U(1)$ under which the gauge singlet components have charges $S_{u}(+1 / 2), S_{d}(-1 / 2), z_{1}(+1), z_{2}(-1)$, equal to the hypercharge of the upper components of the same $S U(3)$ multiplets; it resides mostly in the phase of $S_{u}$ whose VEV dominates the spontaneous symmetry breaking:

$$
S_{u} \simeq f_{u} \exp \left(i \eta / \sqrt{2} f_{u}\right) .
$$

The $\eta$ does not get mass from the $S U(3)$-breaking terms which we so far considered, since they preserve the above $U(1)$; it can however get mass if we add a small $S U(3)$-breaking tadpole

$$
\Delta V_{\text {soft }}=-m_{S}^{3} S_{u}+\text { H.c., }
$$

which gives $m_{\eta}^{2}=m_{S}^{3} / f$. This term breaks $S_{u} \rightarrow-S_{u}$ symmetry and can be generated radiatively by adding to the superpotential a small term $\Delta W=m^{\prime} T t^{c}$ breaking the same symmetry:

$$
m_{S}^{3} \simeq-\frac{3 y}{2 \pi^{2}} m^{\prime} m_{Q}^{2} \ln \frac{M_{\mathrm{GUT}}}{F} .
$$

It should be noticed however that the discussed axion, even if exactly massless, would not be in conflict with experiment [11], since it couples very weakly to the ordinary matter (such a coupling 
could for example proceed via mixing with heavy fermions which are needed to implement the $S U(3)$ symmetry in the first and second generations).

Our last comment concerns the impact of the triplets $T_{1}, T_{2}$ on the precision electroweak observables. According to Eq. (2.4) they get VEVs, $T_{i}^{11} \simeq f_{Z i} \frac{v^{2}}{f^{2}}$. Since according to our discussion below Eq. (2.12) we need $f_{Z 1}<0.1 f$ (and $f_{Z 2}$ is even smaller), the contribution of the triplets to the $\rho$ parameter is sufficiently suppressed:

$$
\delta \rho=-2 \frac{\left(T_{1}^{11}\right)^{2}}{v^{2}}, \quad|\delta \rho|<10^{-4}\left(\frac{1.7 \mathrm{TeV}}{f}\right)^{2} .
$$

We conclude that Model I is a fully consistent example of a supersymmetric model with a Higgs doublet as a Goldstone boson of extended global symmetry, perturbative up to the GUT scale and with large $\tan \beta$. Its phenomenology is similar to phenomenology of the MSSM in the decoupling limit, but finetuning in the Higgs potential is diminished by at least a factor 10 compared to MSSM. The lightest Higgs mass is expected to be just above $115 \mathrm{GeV}$, with stop around $800 \mathrm{GeV}$, and the new top quark above $3 \div 4 \mathrm{TeV}$, probably unreachable at the LHC. However, stabilization of the $S U(3)$ breaking potential requires new states. The extended scalar sector of Model I is unlikely to manifest itself at the LHC, since these particles are expected to be quite heavy (apart from the decoupled axion $\eta$ ), while their couplings to $W W$ and $t \bar{t}$ are suppressed due to large $\tan \beta$ and $f / v$ ratio.

Among new fermions, there are those with the same quantum numbers of MSSM chargino and neutralino states. However, the mixing between MSSM states and these new fermions is small because $f_{Z i} \ll f$ and the mass eigenstates remain almost MSSM-like. The new mass eigenstates, including $\tilde{T}^{ \pm \pm}$and $\tilde{z}_{i}$ 's, are almost degenerate in mass, with masses $m \approx \mu_{Z} \approx 200 \mathrm{GeV}$. The details of the mass spectrum depend on the details of the mixing. Thus we expect new light fermions doubling the MSSM states, but the best chance to see a trace of the $S U(3)$ structure is probably the doubly charged Higgsino. At the LHC, this double-chargino $\tilde{T}^{ \pm \pm}$will be pairproduced via the Drell-Yan process. It will most likely undergo a chain decay into $\tilde{T}^{ \pm}$and $W$ and finally into the lightest neutralino and two same sign $W$ bosons. Because of an approximate degeneracy of the mass spectra the decay products are likely to be off-shell. The double-chargino is likely to be long-lived and the decay vertex may be displaced. In any case, we expect events with two opposite-hemisphere pairs of same-sign leptons and missing transverse energy in the final state. We are not aware of experimental studies for signals of this typ 5 . By analogy with DrellYan chargino-neutralino searches, we can optimistically estimate the LHC integrated luminosity required for the discovery of $\tilde{T}^{ \pm \pm}$to be $O(100) \mathrm{fb}^{-1}$. The fermionic neutral singlets $\tilde{z}_{i}$ 's may also be phenomenologically interesting. Depending on the details of the mass spectrum one of them may be the LSP and a potential candidate for dark matter particle. Our model provides then a concrete example of a spectrum going beyond the MSSM spectrum. More detailed phenomenological study of such and similar spectra are of experimental interest but beyond the scope of this paper.

\footnotetext{
${ }^{5}$ In [18 doubly charged Higgsinos with a significant coupling to leptons were considered, so that a dominant decay mode is into slepton-lepton pairs, with the slepton subsequently decaying into a lepton plus neutralino. This gives rise to a practically background-free same-sign, same-flavor lepton pair and missing energy signature. Such couplings in our model would violate the lepton number conservation and are by no means necessary (if allowed at all). Our case is definitely more challenging experimentally.
} 


\section{$3 \quad$ Model II}

It has been emphasized in [7] (following an earlier observation in [2]), that in models with extended global symmetry there exists also a mechanism for EWSB and the Higgs boson mass generation based on a tadpole contribution of an $S U(2) \times U(1)$ singlet component of the full scalar multiplet. This mechanism necessarily requires a small value of $f$, to minimize finetuning in the Higgs potential. In turn, this implies large quartic coupling and low UV cutoff. On the other hand, high enough Higgs boson mass can be produced even for moderate $\tan \beta$.

The simplest model achieving stabilization of $S U(3)$ breaking at small $f$ includes two Higgs triplets $\mathcal{H}_{d}$ and $\mathcal{H}_{u}$, same as in Model I, and an $S U(3)$ singlet $N$, with the superpotential

$$
W=\kappa N \mathcal{H}_{u} \mathcal{H}_{d}
$$

As we will see, it generically leads to low values of $\tan \beta$. We must have $\kappa \leq 2$ for the Landau pole to be above $\Lambda_{\mathrm{UV}}=20-30 \mathrm{TeV}$; we will choose $\kappa=2$ in what follows. All RG runnings below are considered from $\Lambda_{\mathrm{UV}}$ down to the Fermi scale, $\log \frac{\Lambda_{\mathrm{UV}}}{M_{\mathrm{SUSY}}} \simeq 3$ for $M_{\mathrm{SUSY}} \sim 1 \mathrm{TeV}$.

The scalar potential reads:

$$
\begin{aligned}
V & =\kappa^{2}\left[\left|\mathcal{H}_{u} \mathcal{H}_{d}\right|^{2}+|N|^{2}\left(\left|\mathcal{H}_{u}\right|^{2}+\left|\mathcal{H}_{d}\right|^{2}\right)\right]+V_{\text {soft }} \\
V_{\text {soft }} & =m_{u}^{2}\left|\mathcal{H}_{u}\right|^{2}+m_{d}^{2}\left|\mathcal{H}_{d}\right|^{2}+m_{N}^{2}|N|^{2}-\left(A^{3} N+m_{3}^{2} \mathcal{H}_{u} \mathcal{H}_{d}+\text { H.c. }\right)
\end{aligned}
$$

The masses $m_{u, d}^{2}, m_{N}^{2}$ need to be positive to avoid runaway. We use $m_{3}^{2}$ to break $S U(3)$ spontaneously, while $A^{3}$ will give a VEV to $N$ and generate an effective $\mu$-term (chargino mass). It is consistent to assume that all terms which are not included at the tree level remain small or vanish. E.g. $A^{\prime} \mathrm{NH}_{1} \mathrm{H}_{2}$ term is generated by gaugino masses,

$$
\delta A^{\prime} \sim \frac{g^{2} \kappa M_{2}}{16 \pi^{2}} \times 3 \sim 0.02 M_{2} .
$$

Possible modifications of the model can be obtained by adding $\mu \mathcal{H}_{u} \mathcal{H}_{d}$ and/or $\kappa F^{2} N$ terms to the superpotential, as well as $N \mathcal{H}_{u} \mathcal{H}_{d}$ term to $V_{\text {soft }}$. These modifications lead to models of comparable "quality", and we will not consider them.

Minimization of the potential (3.1) in the gauge singlet direction gives

$$
\begin{gathered}
\left\langle\left|\mathcal{H}_{u, d}\right|\right\rangle \equiv f_{u, d}, \quad f^{2} \equiv f_{u}^{2}+f_{d}^{2}=\frac{m_{3}^{2}-\mu_{u} \mu_{d}}{\kappa^{2} \sin \beta \cos \beta}, \quad \tan \beta \equiv \frac{f_{u}}{f_{d}}=\frac{\mu_{d}}{\mu_{u}}, \\
\mu_{u, d}^{2} \equiv m_{u, d}^{2}+\mu^{2}, \quad \mu \equiv \kappa f_{N}, \quad\langle|N|\rangle \equiv f_{N}=\frac{A^{3}}{m_{N}^{2}+\kappa^{2} f^{2}}
\end{gathered}
$$

where we continue using notation of section 2 for the components of $\mathcal{H}_{d}$ and $\mathcal{H}_{u}$.

As in Model I, spontaneous breaking of $S U(3)$ to $S U(2)$ generates five Goldstone bosons, a doublet $H$ and an axion. $S U(3)$ symmetry must then be broken also explicitly, to get a potential for $H$, which will break electroweak symmetry. In this model the presence of the soft term $m_{3}^{2}$ results in nonvanishing VEVs $f_{u, d, N}$ breaking the global $S U(3)$. To avoid any risk of destabilization of the $S U(3)$ breaking potential by a negative $m_{u}^{2}$, we keep the top-stop sector as in MSSM. Thus, 
$S U(3)$ is explicitly broken by the top-stop sector. The standard RG running from $\Lambda_{\mathrm{UV}}$ generates a negative contribution to the Higgs mass squared

$$
-\delta m_{H}^{2}\left|H_{u}\right|^{2} \equiv-\delta m_{H}^{2}\left(\left|\mathcal{H}_{u}\right|^{2}-\left|S_{u}\right|^{2}\right)
$$

Another source of the explicit $S U(3)$ breaking is a tadpole contribution $m_{S}^{3} S_{u}$, which, we assume, is generated by strong dynamics at $\Lambda_{\mathrm{UV}} 6$. Making use of (3.3), we can write the full $S U(3)$ breaking potential as a function of $S_{u}$ :

$$
\Delta V=m_{H}^{2}\left|S_{u}\right|^{2}-\left(m_{S}^{3} S_{u}+\text { H.c. }\right) \text {, }
$$

which has the clear advantage of completely decoupling the $S U(3)$-symmetric potential minimization and vacuum disalignment. In this parametrization, we view the $\left|\mathcal{H}_{u}\right|^{2}$ contribution in (3.3) as a renormalization of $m_{u}^{2}$ parameter in (3.1). Minimizing the CP-even part of (3.4), we find the VEV of $S_{u}$ :

$$
\left\langle\left|S_{u}\right|\right\rangle=\frac{m_{S}^{3}}{m_{H}^{2}},
$$

where we have to assume that the found minimum satisfies $\left|S_{u}\right|<f_{u}$, otherwise the true minimum will be at $S_{u} \simeq \pm f_{u}$ with no EWSB. On the other hand, $\left|S_{u}\right|<f_{u}$ means vacuum disalignment, with the Higgs VEV

$$
\left\langle\left|H_{u}\right|^{2}\right\rangle \equiv v_{u}^{2}=f_{u}^{2}-\left\langle\left|S_{u}\right|^{2}\right\rangle
$$

and the EWSB scale given by

$$
v^{2}=v_{u}^{2}+v_{d}^{2}=f^{2}\left[1-\left(\frac{m_{S}^{3}}{f_{u} m_{H}^{2}}\right)^{2}\right] .
$$

We see that $v \ll f$ can be obtained only at the price of finetuning the ratio $m_{S}^{3} / f_{u} m_{H}^{2}$ to 1 . To illustrate this finetuning more clearly, we can express (3.4) as a function of $\left|H_{u}\right|$ by expanding $S_{u}=\sqrt{\left|f_{u}\right|^{2}-\left|H_{u}\right|^{2}}$, which is a good approximation for $v \ll f$ :

$$
\Delta V \simeq \text { const }-\left(m_{H}^{2}-m_{S}^{3} / f_{u}\right)\left|H_{u}\right|^{2}+\lambda\left|H_{u}\right|^{4}+\ldots, \quad \lambda=\frac{m_{S}^{3}}{4 f_{u}^{3}} .
$$

We now see the origin of finetuning: it appears since we are canceling the $O\left(f^{2}\right)$ Higgs mass term with the quadratic term appearing in the expansion of $\sqrt{f_{u}^{2}-\left|H_{u}\right|^{2}}$, taking advantage of the non-linear structure of the $\sigma$-model.

The finetuning discussed above can be quantified by means of the usual logarithmic derivative, or by measuring the portion of the uniformly distributed parameter space satisfying $v \leq 174 \mathrm{GeV}$; we get $[7]$

$$
\mathrm{FT} \simeq \frac{2 f^{2}}{v^{2}}
$$

\footnotetext{
${ }^{6}$ Perturbative origin of the tadpole could be engineered if desired by breaking $S_{u} \rightarrow-S_{u}$ symmetry in the superpotential, similarly to the generation of (2.16) in Model I.

${ }^{7}$ This finetuning does not increase even if $m_{H}$ and $m_{S}$ are scaled up, because the Higgs quartic $\lambda$ in (3.7) and, correspondingly, the Higgs mass (see below), increases in the same limit, "improving naturalness" in the sense of [15].
} 
As a reference value we fix $f=350 \mathrm{GeV}$, corresponding to $O(10) \%$ finetuning 8 .

Other potential sources of finetuning in Model II are related to the RG running of the $S U(3)$ symmetric potential parameters. According to Eq. (3.2) to avoid large cancellations in the potential for $S U(3)$ breaking, we must have

$$
\mu_{u} \mu_{d} \lesssim \kappa^{2} f^{2} / 2
$$

i.e. effectively

$$
\mu^{2}, m_{u}^{2}, m_{d}^{2} \lesssim \kappa^{2} f^{2} / 4
$$

As mentioned above, the $\left|\mathcal{H}_{u}\right|^{2}$ in Eq. (3.3) effectively renormalizes $m_{u}^{2}$; naturalness thus requires that

$$
\delta m_{H}^{2}=\frac{3}{4 \pi^{2}} y_{t}^{2} m_{\tilde{t}}^{2} \log \frac{\Lambda_{\mathrm{UV}}}{M_{\mathrm{SUSY}}} \sim \frac{m_{\tilde{t}}^{2}}{4 \sin ^{2} \beta} \lesssim m_{u}^{2} \lesssim \kappa^{2} f^{2} / 4
$$

Thus we get

$$
m_{\tilde{t}} \lesssim \sin \beta \kappa f .
$$

Finally, we discuss the Higgs boson mass. The same expansion of the square root which allows us to finetune $v \ll f$ in (3.7), also generates a Higgs quartic $\lambda$. For small $\tan \beta$ this quartic easily dominates the standard $D$-term quartic; as a result the Higgs boson mass is solely determined by the soft terms and the coupling $\kappa$. Taking into account the $\sigma$-model wavefunction suppression and also using the exact expression of the Higgs potential (3.4) instead of expanding in $H_{u}$, we find the Higgs boson mass

$$
m_{h}=\sin \beta\left(m_{H} / f\right) v .
$$

If we assume that $m_{H}^{2}$ is entirely generated by stop loops we get

$$
m_{h}=\frac{v}{2} \frac{m_{\tilde{t}}}{f}
$$

and thus $m_{\tilde{t}} \simeq \sqrt{2} f$ for $m_{h} \simeq 120 \mathrm{GeV}$, consistently with the constraint (3.11) on $m_{\tilde{t}}$ for low $\tan \beta$. The model then predicts a light Higgs boson since larger values of $m_{\tilde{t}} / f$ are inconsistent with the constraint (3.11).

We will discuss phenomenology of Model II for $f=350 \mathrm{GeV}, 1 \leq \tan \beta \leq 2$, and $\kappa=2$.We choose the potential parameters as follows $(t \equiv \tan \beta)$ :

$$
\begin{aligned}
& \mu^{2}=m_{u}^{2}=\frac{\kappa^{2} f^{2} / 2}{1+t^{2}}, \quad m_{d}^{2}=\frac{2 t^{2}-1}{t^{2}+1} \kappa^{2} f^{2} / 2 \\
& m_{3}^{2}=\frac{2 \kappa^{2} f^{2}}{t+t^{-1}}, \quad m_{N}^{2}=6 m_{u}^{2}, \quad A^{3}=\left(m_{N}^{2}+\kappa^{2} f^{2}\right) \frac{\mu}{\kappa}
\end{aligned}
$$

which is consistent with naturalness and produces a minimum of the potential at the given values of $f$ and $\tan \beta$. We see that Higgsinos are expected to be light, $\mu \sim 100 \div 200 \mathrm{GeV}$, for $f=350$ $\mathrm{GeV}$.

\footnotetext{
${ }^{8}$ Comparing with [7, notice a factor $\sqrt{2}$ difference in normalization of $f$ resulting from the change from real to complex fields. Our $f=350 \mathrm{GeV}$ gives the same finetuning as $f \simeq 500 \mathrm{GeV}$ in [7].
} 
Furthermore, since $f$ is small, phenomenology of the model is strongly influenced by its nonlinear structure. We recall that in the $\sigma$-model approximation

$$
\mathcal{H}_{u}=f_{u}\left(\begin{array}{l}
\frac{H}{|H|} \sin \left(\frac{|H|}{f}\right) \\
\cos \left(\frac{|H|}{f}\right)
\end{array}\right)
$$

and similarly for $\mathcal{H}_{d}\left(|H|=\sqrt{H^{\dagger} H}\right)$. We also can parametrize the Higgs doublet $H$ nonlinearly:

$$
H=\Sigma\left(\begin{array}{c}
0 \\
\bar{v}+h / \sqrt{2}
\end{array}\right)
$$

where $\Sigma=e^{i T^{a} G^{a} / v}$ is the pion field containing the Goldstone bosons eaten by the W and Z. The fields are canonically normalized. The true electroweak scale reads $v=f \sin (\bar{v} / f)$. Additional heavy scalar modes describe deviation from the $\sigma$-model structure (3.12). For instance, fluctuations in the radial directions can be introduced by replacing

$$
f_{u, d} \rightarrow f_{u, d}+s_{u, d} / \sqrt{2} .
$$

We are interested in the couplings of the scalars $h, s_{u}, s_{d}$ to the vector boson pairs. By the equivalence theorem, these couplings can be obtained from the kinetic part of the Lagrangian for $\mathcal{H}_{u}$ and $\mathcal{H}_{d}$ inserting (3.13) into (3.12). The couplings of $s_{u, d}$ are found using (3.14). We get:

$$
\mathcal{L}=v^{2}\left|D_{\mu} \Sigma\right|^{2}+\sqrt{2} v\left|D_{\mu} \Sigma\right|^{2}\left[\cos (\bar{v} / f) h+\frac{v}{f}\left(\cos \beta s_{u}+\sin \beta s_{d}\right)\right]
$$

We see that the $h$ coupling to pions, and hence to $W W$, is suppressed by $\cos (\bar{v} / f)=\left(1-v^{2} / f^{2}\right)^{1 / 2}$, and $h$ unitarizes $W W$ amplitude only partially. Unitarization is completed by the exchange of the heavy scalars. The $s_{u, d} W W$ couplings appear because the radial directions obtain nonzero projection on the first two components of $\mathcal{H}_{u, d}$ when expanded around $v \neq 0$.

The fields $s_{u}$ and $s_{d}$ are not mass eigenstates. In the mass matrix, they mix with each other and also with the radial excitation of $N$. Thus, three heavy mass eigenstates complete the unitarization of $W W$ scattering. Denoting the mass eigenstates by $S_{i}, i=1,2,3$, e.g. for $f=350 \mathrm{GeV}, \kappa=2$ and $\tan \beta=2$ we get $m_{S_{i}} \simeq(290,850,1000) \mathrm{GeV}$. The cubic $W W$-scalar interaction Lagrangian in this case is given by

$$
\begin{aligned}
\mathcal{L} & =g_{W W h}^{\mathrm{SM}}\left[\cos (\bar{v} / f) h+(v / f) c_{i} S_{i}\right] W_{\mu}^{2}, \\
c & \simeq(0.86,-0.13,-0.5), \quad c_{i}^{2}=1,
\end{aligned}
$$

so that the $W W$ scattering is fully unitarized above $m_{S 3}$.

Other important couplings are the $S_{i} \bar{t} t$ couplings as they determine the production rate of these scalars via the gluon fusion. They originate from the term

$$
y_{t} \frac{v}{f} \frac{s_{u}}{\sqrt{2}} t \bar{t}, \quad y_{t} \equiv \frac{m_{t}}{v \sin \beta},
$$

which appears similarly to the $s_{u, d} W W$ couplings discussed above. Due to the $v / f$ coupling suppression, production rates of the heavy scalars via the gluon fusion, as well as via the vector boson 
fusion, will be suppressed by at least one order of magnitude with respect to the corresponding production rates for the SM Higgs boson of the same mass. Nevertheless, at least the lightest of these heavy scalars should be quite easy to discover at the LHC in the gold-plated decays $S_{1} \rightarrow Z Z \rightarrow 4 l$.

Apart from the radial modes (3.14), another interesting heavy mode is a longitudinal fluctuation orthogonal to the pseudo-Goldstone mode:

$$
H_{u}=\cos \beta H_{1}, \quad H_{d}=-\sin \beta H_{1} .
$$

The $S U(2)$ doublet $H_{1}$ does not get a VEV and is decoupled from the vector boson pairs; it is analogous to the heavy MSSM doublet in the decoupling limit. It describes a degenerate heavy multiplet $\left(H^{ \pm}, H^{0}, A^{0}\right)$ of mass

$$
m_{H 1}^{2} \simeq \frac{\mu_{u} \mu_{d}}{\sin \beta \cos \beta},
$$

which can be found substituting (3.17) into the scalar potential. By (3.9), we expect $m_{H 1}=$ $O(\kappa f) \sim 700 \mathrm{GeV}$. The neutral members of this multiplet couple to $t \bar{t}$ with strength $\cot \beta$ times the SM Higgs coupling. They will be produced via gluon fusion and will be seen as narrow resonances decaying into $t \bar{t}$ pairs (total width around $30 \mathrm{GeV}$ ). Using the model-independent analysis of [16], we can estimate that $O(10) \mathrm{fb}^{-1}$ of integrated luminosity could be enough for their discovery at the LHC.

Finally, we discuss the effect of the heavy scalars on the electroweak observables. As pointed out in Ref.[7], the relevant parameter is the effective Higgs boson mass, which in our case is given by

$$
m_{\mathrm{EWPT}}=m_{h}\left(\frac{\bar{m}}{m_{h}}\right)^{\frac{v^{2}}{f^{2}}}, \quad \bar{m}=\prod\left(m_{S_{i}}\right)^{c_{i}^{2}},
$$

where the $c_{i}$ are the parameters appearing in the $W W$-scalar interaction Lagrangian (3.16). In our numerical example we get $\bar{m}=400 \mathrm{GeV}$ and $m_{\mathrm{EWPT}}=155 \mathrm{GeV}$ for $m_{h}=115 \mathrm{GeV}$. Thus, $m_{\mathrm{EWPT}}$ is slightly above the $144 \mathrm{GeV} 95 \%$ C.L. limit, but various other supersymmetric contributions can easily compensate its effect in the $(S, T)$ plane.

\section{Conclusions}

We have presented two realistic supersymmetric models with Higgs doublet as Goldstone boson of a spontaneously broken extended global symmetry. Model I is perturbative up to the GUT scale and realizes large $\tan \beta$ scenario, while Model II requires a rather low UV cut-off $(\sim 20 \mathrm{TeV})$ and generically gives low $\tan \beta$. Both models avoid excessive finetuning in the Higgs potential and are in fact motivated by this requirement. Being perturbative up to much higher cut-off than so-called "strongly interacting" models, they do not lead to any serious tension with precision electroweak data. The two models illustrate two different mechanisms for EWSB and the Higgs mass generation. Their experimental signatures are quite different. Clearly, the price for a small finetuning is some complexity (e.g. compared to the MSSM). Our constructions supplement the list of previous proposals for ameliorating the supersymmetric little hierarchy problem. E.g. the Next-to-Minimal Supersymmetric Standard Model easily solves the little hierarchy if its $S H_{u} H_{d}$ 
coupling $\lambda$ is allowed to become strong below the GUT scale (a possibility recently taken to the extreme in [17]). Its predictions are different from Model II as, for instance, it does not predict non-linear effects in the scalar couplings. We will wait and see what experiment tells us. For the moment, the main lesson of our constructions is that the possibility of the Higgs boson as a Goldstone boson in perturbative theories looks equally plausible as in non-perturbative scenarios with low cut-off and actually more predictive.

\section{Acknowledgements}

V.S.R. was supported by the EU under RTN contract MRTN-CT-2004-503369 and ToK contract MTKD-CT-2005-029466.

\section{A Technical details on Model I}

In this appendix we collect some technical details relevant for Model I. It is convenient to normalize all charges and couplings with the UV completion into $S U(3) \times U(1)_{x}$ in mind. For instance $\mathcal{H}_{u}$ has $S U(3) \times U(1)_{x}$ quantum numbers $3_{1 / 3}$,

$$
D \mathcal{H}_{u}=\left(W^{a} T^{a}+1 / 3 B_{x}\right) \mathcal{H}_{u} .
$$

The unbroken generator is $Y=\frac{1}{\sqrt{3}} T^{8}+X$. The $S U(3)$ gauge coupling $g$ coincides at the scale $F$ with the $S U(2)$ gauge coupling $g_{2}$. The $U(1)_{y}$ coupling $g_{y}$ at $F$ is:

$$
g_{y}=\frac{g g_{x}}{\sqrt{g^{2}+g_{x}^{2} / 3}} .
$$

Numerical values of $g$ and $g_{x}$ are 0.65 and 0.37 respectively. The RG equation for $m_{3}^{2}$ in Eq. (2.2) valid above $F$ reads

$$
16 \pi^{2} \frac{d m_{3}^{2}}{d \log \Lambda}=-\frac{16}{3} g^{2} M \mu+U(1)_{x} \text {-gaugino contribution. }
$$

Here $M$ is the $S U(3)$-symmetric gaugino mass. For the running from the GUT scale we get

$$
\delta m_{3}^{2} \sim 0.4 M \mu .
$$

From (2.3), the natural value of $\tan \beta$ is $m_{d}^{2} / \delta m_{3}^{2}$. We see that $\tan \beta=O(10)$ is naturally allowed, provided that $m_{d}$ is a factor of a few larger than $M, \mu$. Running from $F$ down generates only $H_{u} H_{d}$ coefficient which is much smaller since the running is very short.

Other mass parameters whose running is of interest for the model are $m_{u}^{2}$ and $m_{Z 2}^{2}$. For $\log M_{\mathrm{GUT}} / F \sim 30$ one gets

$$
\begin{aligned}
\delta m_{u}^{2} & =\frac{1}{16 \pi^{2}} 6 y^{2}\left(m_{Q}^{2}+m_{T^{c}}^{2}+m_{u}^{2}\right) \times 30+\frac{1}{16 \pi^{2}} 16 \lambda^{2}\left(m_{u}^{2}+m_{Z 2}^{2}\right) \times 30 \\
\delta m_{Z 2}^{2} & =\frac{1}{16 \pi^{2}} 4 \lambda^{2}\left(m_{u}^{2}+m_{Z 2}^{2}\right) \times 30
\end{aligned}
$$


The dominant contribution comes from the term proportional to the Yukawa coupling. It makes $m_{u}^{2}$ negative and breaks the global $S U(3)$ symmetry radiatively.

Finally, we discuss the perturbativity constraint up to the GUT scale on the couplings $\lambda$ and $y$. The RG equations read:

$$
\begin{aligned}
& 16 \pi^{2} \frac{d y}{d \log \Lambda}=y\left(7 y^{2}+8 \lambda^{2}-\frac{16}{3}\left(g_{3}^{2}+g^{2}\right)-O\left(g_{x}^{2}\right)\right) \\
& 16 \pi^{2} \frac{d \lambda}{d \log \Lambda}=\lambda\left(18 \lambda^{2}+6 y^{2}-12 g^{2}-\frac{4}{3} g_{x}^{2}\right)
\end{aligned}
$$

where $g_{3}$ is the strong coupling constant. One can check that the safe range for values of the couplings at the scale $F$ is $y \lesssim 1.2, \lambda \lesssim 0.3$.

\section{References}

[1] D. B. Kaplan and H. Georgi, "SU(2) X U(1) Breaking By Vacuum Misalignment," Phys. Lett. B 136, 183 (1984). D. B. Kaplan, H. Georgi and S. Dimopoulos, "Composite Higgs Scalars," Phys. Lett. B 136, 187 (1984).

[2] K. Agashe, R. Contino and A. Pomarol, "The minimal composite Higgs model," Nucl. Phys. B 719, 165 (2005) [arXiv:hep-ph/0412089].

[3] R. Contino, L. Da Rold and A. Pomarol, "Light custodians in natural composite Higgs models," Phys. Rev. D 75, 055014 (2007) arXiv:hep-ph/0612048.

[4] R. Contino, T. Kramer, M. Son and R. Sundrum, "Warped/Composite Phenomenology Simplified," JHEP 0705, 074 (2007) |arXiv:hep-ph/0612180|.

[5] G. F. Giudice, C. Grojean, A. Pomarol and R. Rattazzi, "The strongly-interacting light Higgs," JHEP 0706, 045 (2007), arXiv:hep-ph/0703164.

[6] A. Falkowski, S. Pokorski and J. P. Roberts, "Modelling strong interactions and longitudinally polarized vector boson scattering," JHEP 0712, 063 (2007) arXiv:0705.4653 [hep-ph]].

[7] R. Barbieri, B. Bellazzini, V. S. Rychkov and A. Varagnolo, "The Higgs boson from an extended symmetry," Phys. Rev. D 76, 115008 (2007) arXiv:0706.0432 [hep-ph].

[8] C. Csaki, J. Heinonen, M. Perelstein and C. Spethmann, "A Weakly Coupled Ultraviolet Completion of the Littlest Higgs with T-parity," arXiv:0804.0622 [hep-ph].

[9] A. Birkedal, Z. Chacko and M. K. Gaillard, "Little supersymmetry and the supersymmetric little hierarchy problem," JHEP 0410, 036 (2004) arXiv:hep-ph/0404197.

[10] P. H. Chankowski, A. Falkowski, S. Pokorski and J. Wagner, "Electroweak symmetry breaking in supersymmetric models with heavy scalar superpartners," Phys. Lett. B 598, 252 (2004) arXiv:hep-ph/0407242.

[11] Z. Berezhiani, P. H. Chankowski, A. Falkowski and S. Pokorski, "Double protection of the Higgs potential," Phys. Rev. Lett. 96, 031801 (2006) arXiv:hep-ph/0509311.

[12] T. Roy and M. Schmaltz, "Naturally heavy superpartners and a little Higgs," JHEP 0601, 149 (2006) arXiv:hep-ph/0509357. 
[13] C. Csaki, G. Marandella, Y. Shirman and A. Strumia, "The super-little Higgs," Phys. Rev. D 73, 035006 (2006) arXiv:hep-ph/0510294.

[14] A. Falkowski, S. Pokorski and M. Schmaltz, "Twin SUSY," Phys. Rev. D 74, 035003 (2006) arXiv:hep-ph/0604066].

[15] R. Barbieri, L. J. Hall and V. S. Rychkov, "Improved naturalness with a heavy Higgs: An alternative road to LHC physics," Phys. Rev. D 74, 015007 (2006) arXiv:hep-ph/0603188].

[16] V. Barger, T. Han and D. G. E. Walker, "Top Quark Pairs at High Invariant Mass - A ModelIndependent Discriminator of New Physics at the LHC," Phys. Rev. Lett. 100, 031801 (2008) arXiv:hep-ph/0612016.

[17] R. Barbieri, L. J. Hall, Y. Nomura and V. S. Rychkov, "Supersymmetry without a light Higgs boson," Phys. Rev. D 75, 035007 (2007) arXiv:hep-ph/0607332.

[18] D. A. Demir, M. Frank, K. Huitu, S. K. Rai and I. Turan, "Signals of Doubly-Charged Higgsinos at the CERN Large Hadron Collider," arXiv:0805.4202 [hep-ph]. 